\title{
ANÁLISE DO DESEMPENHO MOTOR ESPORTIVO DE CRIANÇAS E ADOLESCENTES COM TRANSTORNO DO ESPECTRO AUTISTA
}

\author{
SPORTIVE MOTOR PERFORMANCE ANALYSIS OF CHILDREN AND
}

ADOLESCENTS WITH AUTISTIC SPECTRUM DISORDER

\author{
Felipe Soares Pereira ${ }^{1}$ \\ Josiane Fujisawa Filus de Freitas ${ }^{2}$
}

\begin{abstract}
RESUMO: O objetivo do presente estudo foi analisar o desempenho motor esportivo de crianças e adolescentes com Transtorno do Espectro Autista, uma síndrome do neurodesenvolvimento que afeta principalmente áreas de comportamento e comunicação social. A pesquisa é considerada descritiva e foi composta por 6 indivíduos com idades entre 8 e 13 anos, alunos de um projeto de educação física voltado a autistas da cidade de Dourados-MS. Utilizou-se os testes de velocidade de deslocamento e força explosiva dos membros inferiores do Projeto Esporte Brasil (PROESP-BR) e observações de um período de 10 meses de aulas do projeto. Todos os sujeitos apresentaram resultado considerado "fraco" nos testes, porém algumas questões relativas ao teste e à deficiência devem ser consideradas, justificando o desempenho dos avaliados. Concluiu-se que a atividade física é um importante mecanismo de desenvolvimento motor, cognitivo e social dos autistas e, portanto, a presença do profissional de educação física é fundamental na vida dessas pessoas.
\end{abstract}

Palavras-chave: Transtorno do Espectro Autista. Educação Física. PROESP-Brasil.

\begin{abstract}
The objective of the present study was to analyze the sports motor performance of children and adolescents with Autistic Spectrum Disorder, a neurodevelopment syndrome that mainly affects areas of behavior and social communication. The research is descriptive qualitative approach considered and was composed of 6 individuals aged between 8 and 13 years, students of a physical education project focused on autistic city of Dourados-MS. We used the speed tests and explosive force of the lower limbs Sport Brazil Project (PROESP-BR) and observations of a period of 10 months of classes in the project. All subjects presented a result considered "weak" in testing, however some issues related to testing and disability should be considered, justifying the assessed performance. It was concluded that physical activity is an important mechanism of motor, cognitive and social development of autistic and, therefore, the presence of professional physical education is fundamental in the life of these people.
\end{abstract}

Keywords: Autistic Spectrum Disorder. Physical Education. PROESP-Brazil.

\footnotetext{
${ }^{1}$ Graduado em Licenciatura em Educação Física pela Universidade Federal da Grande Dourados (UFGD). Email: fspereira95@hotmail.com

${ }^{2}$ Docente do Curso de Educação Física da Universidade Federal da Grande Dourados. E-mail: josianefffreitas@ufgd.edu.br
} 


\section{Introdução}

O Transtorno do Espectro Autista (TEA) é um distúrbio do neurodesenvolvimento que apresenta características como prejuízo na comunicação e interação social e padrões restritos e repetitivos de comportamento, interesses ou atividades (DSM-V, 2013). Sua primeira definição foi dada por Leo Kanner, em 1943, quando estudou 11 casos e constatou tais características nos pacientes; e um ano depois, o pediatra austríaco Hans Asperger descreveria quatro crianças em situações semelhantes (KLIN, 2006).

Diversos estudos, conforme apontado por Gadia, Tuchman e Rotta (2004), buscaram a obtenção de respostas e avanços, no entanto, tal síndrome ainda permanece com causa desconhecida. Contudo, mesmo não havendo origem e consequentemente cura, o autismo possui tratamento que, de acordo com as necessidades individuais de cada sujeito, envolve equipe multidisciplinar que pode ser formada por psicólogos, terapeutas ocupacionais, fonoaudiólogos, educadores e demais especialistas. Aqui, abordaremos uma área ainda pouco conhecida quando se trata da intervenção desta população: a educação física.

A atividade física tem se revelado importante ferramenta de auxílio na evolução de sujeitos com as mais variadas deficiências. Alguns pesquisadores relataram esses progressos, como é o caso de Poeta e Rosa Neto (2005). A intervenção motora realizada no estudo verificou considerável mudança no desenvolvimento motor de uma criança com Transtorno de Déficit de Atenção e Hiperatividade (TDAH), que assim como o autismo, é enquadrado como transtorno do desenvolvimento, e tem como características principais o excesso de distração e agitação, sendo elas prejudiciais nas tarefas diárias e no desenvolvimento (DSM-V, 2013). Seguindo a mesma linha, Rosa Neto et al. (2013) avaliaram os efeitos da intervenção motora em uma criança autista de 9 anos de idade e constataram avanço significativo nas áreas de motricidade fina e global, equilíbrio e esquema corporal. Lourenço et al. (2016) também contribuem à literatura com um estudo onde mostram a eficácia de um programa de trampolins para 17 crianças com TEA de 4 a 10 anos, apontando melhorias em áreas como coordenação, equilíbrio, velocidade e agilidade.

Agregando à temática "exercício físico e autismo", é relevante também citar as colaborações científicas de Krüger et al. O primeiro estudo (2016a), averiguou os obstáculos da prática de atividade física de um total de 73 crianças autistas com idades entre 4 e 10 anos, de uma cidade do Rio Grande do Sul. Por meio de respostas de questionários preenchidos por seus pais ou responsáveis, chegou-se à conclusão da necessidade de políticas públicas referentes à promoção da educação física para esse grupo. E a segunda pesquisa (2016b), analisou as consequências das atividades rítmicas no desenvolvimento de 10 crianças entre 5 e 10 anos com 
a mesma síndrome. Foram usados questionários para averiguar a situação comportamental das crianças antes e após 14 semanas de práticas, observando-se melhoras nos aspectos de comunicação e interação social e comportamentos repetitivos. Tais atividades revelaram-se interessante mecanismo de evolução corporal e social dessas pessoas.

Os trabalhos acima citados, idem outros, além de apontarem resultados positivos da prática de exercícios físicos para crianças e jovens com transtornos do desenvolvimento, revelaram que os mesmos apresentam atraso motor em relação às pessoas típicas, e no caso dos autistas, esses déficits são identificados desde os primeiros anos de vida, demonstrando uma necessidade de intervenção precoce nesse público (LANDA; MAYER; 2006; NADEL; POSS, 2006; CHAWARSKA et al., 2007; JASMIN et al., 2008 apud ROSA NETO et al., 2013). Dado isso, o estímulo da motricidade durante a infância demonstra importante relevância para aprimorar aspectos motores, cognitivos e sociais desses indivíduos. E tal incentivo muitas vezes poderia ser dado também pelo professor de educação física escolar, visto que na escola é onde os mesmos têm um contato inicial com a atividade física. Porém, a realidade da inclusão desses alunos nas aulas de educação física é distante da ideal e isso se dá por diversos fatores.

A partir do ano de 2012, com a Lei n 12.764 (BRASIL, 2012), o Transtorno do Espectro do Autismo passa a ser enquadrado como deficiência, tornando os autistas detentores dos mesmos direitos das demais pessoas com deficiências. Um desses direitos, previstos na Constituição Federal (BRASIL, 1988) e Lei de Diretrizes e Bases da Educação Nacional (LDB) (BRASIL, 1996), é o acesso à educação, preferencialmente na rede regular de ensino, no qual lhes é assegurado atendimento educacional especializado, com metodologias e estratégias pedagógicas diferenciadas, de forma que atendam às suas especificidades. Esses documentos são de suma importância para a educação inclusiva brasileira, pois contribuem para um aumento significativo - porém gradativo - de alunos com deficiência nas escolas comuns. Todavia, apesar de um grande avanço, o processo de inclusão desses estudantes nas aulas ainda é insatisfatório.

Obstáculos impedem os professores de garantirem uma melhor efetivação da educação inclusiva no Brasil. Na área da educação física não é diferente. De acordo com Fiorini e Manzini (2014, p. 400):

[...] as dificuldades encontradas para atender a demanda da inclusão educacional de alunos com deficiência advêm de diferentes fatores: formação, questão administrativo-escolar, alunos, diagnóstico, família, recurso pedagógico, estratégia de ensino e da área Educação Física.

Partindo deste princípio, os empecilhos apresentados por esses professores se confirmam no que se refere ao ensino de crianças e jovens com autismo, dado que o transtorno 
se manifesta de forma diferente em cada caso. Estudos recentes mostram essas dificuldades, como é o caso de Fiorini e Manzini (2016), que apesar de constatarem situações de sucesso, apontaram grandes entraves por parte dos professores na educação de alunos autistas. Cenários como: orientações somente verbais, informações complexas e uso de expressões ambíguas, foram citadas pelos autores como sendo atitudes realizadas pelos educadores que dificultam a aprendizagem desses sujeitos.

Souza e Assis (2014, p. 6) relataram que "o problema investigado é muito maior do que se pensa", quando desenvolveram uma pesquisa junto a professores de educação física de uma cidade de Goiás a fim de verificar a relação dos mesmos com os alunos autistas e constataram que a inclusão desses alunos ainda é equidistante, posto que houve "mais limites do que propostas e possibilidades da parte desses professores" (Ibid., p. 10). Através de análise com esses profissionais, foi possível perceber que as falhas são as mais diversas e se iniciam desde a concepção de diferença pela sociedade e perpassam pelo conhecimento superficial do transtorno, formação inicial e continuada dos professores com pouca capacitação no assunto e ineficiência das atuais políticas públicas de inclusão (SOUZA; ASSIS, 2014).

Perante o atual contexto, é preciso debater a respeito de metodologias que possibilitem a inclusão de pessoas com TEA não somente na escola, como fora dela também, especialmente na área da educação física que é o foco do estudo, bem como os fatores que contribuem para a dificuldade em se incluir os mesmos na sociedade.

Devido a ausência de estudos na área do comportamento motor de crianças autistas apontada por Rosa Neto et al. (2013), observa-se a necessidade de melhor investigação da temática. A avaliação motora em sujeitos com esse transtorno pode ser feita através de testes que indicarão os déficits na parte motora dos mesmos, e é de suma importância para um melhor trabalho dos profissionais da área, que assim poderão realizar as intervenções necessárias a partir das possíveis dificuldades constatadas (SOARES; CAVALCANTE NETO, 2015). Um desses métodos avaliativos é o Projeto Esporte Brasil (PROESP-BR), que foi selecionado para o presente estudo.

O PROESP-BR (2016) “é um sistema de avaliação da aptidão física relacionada à saúde e ao desempenho esportivo de crianças e adolescentes no âmbito da educação física escolar e do esporte educacional" (GAYA; GAYA, 2016, p. 3). Trata-se de um programa de fácil aplicação que visa analisar de forma simples e prática, o comportamento físico e motor de indivíduos de 7 a 17 anos:

Considerando que a maioria das escolas brasileiras tem carências em sua estrutura física e precária disponibilidade de materiais para as aulas de educação física e esporte educacional, o PROESP desenvolveu uma bateria de 
testes para avaliação de parâmetros de saúde e desempenho motor de muito baixo custo, com o mínimo de materiais sofisticados, de fácil acesso e aplicação, evidentemente, resguardando rigorosamente critérios de validade, fidedignidade e objetividade (GAYA; GAYA, 2016, p. 3-4).

O Projeto também conta com testes adaptados para pessoas com Síndrome de Down (SD), cegas e com deficiência visual. Marques (2008) em sua pesquisa avaliou 2187 indivíduos com Síndrome de Down nos estados de Rio Grande do Sul, Santa Catarina e Paraná, retratando as características desta população e aplicando a bateria de testes do PROESP naqueles com idades entre 10 e 20 anos. O estudo também verificou a qualidade de vida de todos os participantes, associada a condições socioeconômicas, de saúde, independência, prática de atividades físicas, entre outros, chegando-se à conclusão da importância de programas de promoção à saúde a pessoas com $\mathrm{SD}$, contribuindo para um melhor desempenho motor e estilo de vida saudável. Diante disso

[...] desenvolveu-se um programa de acompanhamento chamado de PRODOWN, que configura-se como um observatório contínuo do perfil das crianças e jovens brasileiras com $\mathrm{SD}$, avaliando o crescimento, desenvolvimento somatomotor e aptidão física relacionada à saúde e ao desempenho motor. Esse programa permitirá organizar um banco de dados da população brasileira com SD, que ofereça a possibilidade de desenvolver estudos epidemiológicos, referentes ao estilo de vida, das relações entre a atividade física, exercício físico e doenças associadas, bem como o perfil da aptidão física. (MARQUES, 2008, p. 5)

O PRODOWN conta com os seguintes testes: medidas de massa corporal, estatura, índice de massa corporal (IMC) e envergadura; flexibilidade com e sem banco; força-resistência (abdominal); força explosiva de membros inferiores (salto horizontal); força explosiva dos membros superiores (arremesso de medicineball); agilidade (teste do quadrado); velocidade de deslocamento (corrida de 20 metros); resistência geral (9 minutos); e se difere do PROESP por não possuir algumas medidas de aptidão física para saúde e apresentar pequenas adaptações referentes à flexibilidade, resistência abdominal e resistência geral.

Buscando habilitar um programa adaptado para pessoas cegas e com baixa visão embasado no PROESP-BR, Diehl (2013) aplicou teste e realizou entrevista com 21 crianças e jovens de 7 a 25 anos cegos ou com baixa visão do estado do Rio Grande do Sul, e posteriormente empregou questionário a professores de educação física com experiência no ramo. Mais 72 indivíduos com as mesmas condições foram alvo, com o intuito de verificar a eficiência do teste. Observou-se a necessidade de avaliações físicas mais eficazes, visto que os atuais testes se revelam não muito confiáveis; e maior incentivo a práticas corporais nesse público, pois muitos apresentaram baixo repertório motor por conta das limitações de sua deficiência e falta de ambientes adequados para a realização dessas atividades. Através de 
aplicação do teste, a pesquisa objetivou legitimar o mesmo, o que foi alcançado através de análise de profissionais da área. O projeto foi intitulado BAF- DV (Bateria de Aptidão Física para Crianças e Jovens com Deficiência Visual) e dispõe dos seguintes testes: medida de massa corporal, medida de estatura, flexibilidade, força e resistência abdominal, força explosiva dos membros superiores, força explosiva dos membros inferiores, agilidade, velocidade e resistência cardiovascular/capacidade cardiorrespiratória. A maioria deles segue as mesmas normas do PROESP, sofrendo apenas algumas mudanças na instrução e outros, algumas adaptações referentes a materiais.

Como observado, os dois estudos acima descritos, mediante adaptação de seus autores, deram origem a ferramentas voltadas a populações específicas, tendo validade e fidedignidade comprovadas. Com isso, os autores além de criarem um interessante instrumento de avaliação, demonstraram a valia desse tipo de análise também para sujeitos com deficiência, o que reforça ainda mais a imprescindibilidade do profissional de educação física na vida de todos os tipos de populações. E, dado a necessidade de se avaliar a atividade motora dos autistas, também é crucial a elaboração de uma bateria de testes específica ao público, já que isso ainda não foi realizado.

Assim sendo, o presente estudo que analisa o desempenho motor esportivo de crianças e adolescentes com TEA, visa trazer contribuições à literatura acerca do assunto, oferecendo um suporte teórico a profissionais, familiares e demais interessados; e trazer reflexões acerca da relevância da atividade física para esse grupo em especial.

\section{Metodologia}

A presente pesquisa é tida como descritiva, que Gil (2002, p. 42) define ter como “objetivo primordial a descrição das características de determinada população ou fenômeno ou, então, o estabelecimento de relações entre variáveis"; com abordagem qualitativa.

Os sujeitos do estudo foram 6 crianças e adolescentes com diagnóstico de Transtorno do Espectro do Autismo, todas do sexo masculino, com idades entre 8 e 13 anos, de um total de 37 alunos $^{3}$ atendidos por um projeto de exercício físico voltado a pessoas com essa condição, organizado por uma instituição em que seus familiares deveriam ser associados.

A associação em que os responsáveis pelos sujeitos da pesquisa são filiados é uma entidade sem fins lucrativos localizada na cidade de Dourados-MS que além de realizar um trabalho de divulgação sobre o autismo para a cidade e a região de forma a diminuir a

${ }^{3}$ Dados fornecidos pela própria associação no período de realização da pesquisa. 
desinformação e preconceito, tem o objetivo de promover instrumentos possibilitem um melhor desenvolvimento e inclusão das pessoas com o transtorno na sociedade, como seminários, reuniões, projetos e atendimentos psicológicos. A instituição se mantém por meio de doações, venda de rifas e afins e durante o período de realização da pesquisa contava com mais de 60 famílias associadas.

Como forma de contribuir para o desenvolvimento desses indivíduos, a mesma desenvolve em parceria com as Secretarias Municipais de Educação, Saúde e Assistência Social, projetos que atendem cerca de 50 crianças e jovens ${ }^{4}$ e também conta com atendimento psicológico aos indivíduos e suas famílias.

Um desses projetos é o de educação física, o qual possui atividades visando o aprimoramento de habilidades como coordenação motora fina e grossa, equilíbrio, lateralidade e direcionalidade, salto, esquema corporal, atenção, força e outras, tendo o intuito de através das práticas corporais, proporcionar aos praticantes a ampliação dos seus repertórios motor, cognitivo e afetivo, de modo que adquiram o domínio do próprio corpo, alcançando autonomia em atividades da vida diária e possibilitando uma melhorar interação com outras pessoas em brincadeiras, jogos e demais contextos sociais. Tendo em vista o foco da pesquisa, tal projeto foi escolhido como foco, que conta com 37 alunos com idades entre 3 e 19 anos ${ }^{5}$, que frequentam aulas uma vez por semana com dia e horário fixos.

Os procedimentos foram divididos em: a) entrega de autorização para o (a) responsável pela instituição incumbida do projeto de educação física para autistas, a fim de liberação para realização da pesquisa; b) entrega do Termo de Consentimento Livre e Esclarecido para os pais das crianças e jovens, bem como a explicação da metodologia da pesquisa; c) aplicação dos testes de força explosiva dos membros inferiores (salto horizontal) e velocidade de deslocamento (corrida de 20 metros), da bateria do PROESP-BR (2016); e d) levantamento de dados a partir dos resultados dos testes e descrição dos participantes quanto ao seu processo evolutivo através de observação durante as aulas no projeto.

Os critérios de participação foram de os avaliados terem diagnóstico de Transtorno do Espectro Autista, suas famílias serem associadas da entidade citada anteriormente, e participarem do projeto de educação física para autistas que a associação desenvolve. Foi adotado como norma de exclusão da pesquisa, ter idade menor que 7 e maior que 17 anos, e não possuir autonomia suficiente para atender aos comandos dos testes e realizá-los sozinho, pois o avaliado tinha que executar as tarefas sem auxílio, não podendo haver interferências.

\footnotetext{
${ }^{4}$ Dados fornecidos pela própria associação no período de realização da pesquisa.
}

${ }^{5}$ Dados fornecidos pela própria associação no período de realização da pesquisa. 
A primeira ferramenta de avaliação utilizada foi a bateria de testes do PROESP-BR, mais especificamente os testes de desempenho motor de velocidade e força explosiva dos membros inferiores, que foram escolhidos devido ao menor grau de complexidade, dado que o indivíduo efetua o teste autonomamente. Como parâmetro de performance, empregou-se as tabelas normativas do programa.

O teste de velocidade de deslocamento consiste em o avaliado, em um percurso de 20 metros sinalizado pelo orientador, se deslocar do início ao final da pista o mais rápido possível. É contabilizado o melhor tempo de duas tentativas. No respectivo teste, o avaliador deu instrução verbal, mostrando o local de partida e de chegada, enfatizando a necessidade de realizar esse trajeto com maior velocidade possível por eles.

No teste de força explosiva dos membros inferiores o participante deve executar um salto na maior distância realizável a partir de uma linha traçada no solo, aterrissando com os dois pés ao mesmo tempo. É estabelecida a maior distância de duas tentativas. No referido teste, o avaliador realizou demonstração do salto e ressaltou o requisito da aterrissagem com os dois pés em simultâneo.

O segundo instrumento da pesquisa foi o roteiro de observação das aulas do projeto, considerando um período de 8 meses do ano de 2016 e dois meses de 2017, analisando o comportamento motor, cognitivo e social dos aprendizes durante as sessões.

Todos os sujeitos foram avaliados individualmente em uma quadra poliesportiva adequada às demandas da pesquisa ${ }^{6}$.

\section{Discussão dos resultados}

Tabela 1 - Desempenho no teste de salto em distância

\begin{tabular}{ccc}
\hline Aluno & Resultado $(\mathbf{c m})$ & Classificação segundo o PROESP-BR \\
\hline $\mathbf{1}$ & 41 & Fraco \\
$\mathbf{2}$ & 34 & Fraco \\
$\mathbf{3}$ & 27 & Fraco \\
$\mathbf{4}$ & 41 & Fraco \\
$\mathbf{5}$ & 50 & Fraco \\
$\mathbf{6}$ & 18 & Fraco \\
\hline
\end{tabular}

Fonte: Dados da pesquisa (2017)

É definido por salto "uma habilidade muito parecida com a corrida, mas que envolve uma fase de voo mais longa na decolagem sobre um pé e aterrissagem no outro" (GALLAHUE; DONNELLY, 2008, p.454) e "geralmente é realizado como uma habilidade única, e não como

\footnotetext{
${ }^{6} \mathrm{O}$ estudo atendeu todos os requisitos éticos necessários, aprovados pelo comitê de ética da Universidade Federal da Grande Dourados (UFGD). A liberação de efetuação da pesquisa foi adquirida por intermédio de autorização da responsável pela associação descrita e de Termo de Consentimento Livre e Esclarecido, assinados pelos pais ou responsáveis das crianças e adolescentes.
} 
uma habilidade repetida" (Ibid., p. 449). No respectivo procedimento, o sujeito realizou duas tentativas de salto horizontal, onde teria que aterrissar com os dois pés ao mesmo tempo ao solo, calculando a melhor marca.

Considerando que a idade dos participantes vai de 8 a 13 anos, segundo a classificação do teste, são considerados como resultado "fraco", valores $<118 \mathrm{~cm}$ para 8 anos e $<159 \mathrm{~cm}$ para 13 anos; e "excelência", >= $166 \mathrm{~cm}$ para 8 anos e >= $217 \mathrm{~cm}$. Como demonstrado na tabela, os alunos alcançaram resultados inferiores para a idade e sexo.

No que se refere à execução do teste, alguns alunos apresentaram dificuldade na aterrissagem com os dois pés, mas os erros foram corrigidos pelos examinadores e as análises prosseguiram sem mais interferências.

Tabela 2 - Desempenho no teste de velocidade de deslocamento

\begin{tabular}{ccc}
\hline Aluno & Resultado (seg.) & Classificação segundo o PROESP- \\
& & BR \\
\hline $\mathbf{1}$ & 5,46 & Fraco \\
$\mathbf{2}$ & 6,89 & Fraco \\
$\mathbf{3}$ & 5,27 & Fraco \\
$\mathbf{4}$ & 5,46 & Fraco \\
$\mathbf{5}$ & 4,79 & Fraco \\
$\mathbf{6}$ & 7,45 & Fraco \\
\hline
\end{tabular}

Fonte: Dados da pesquisa (2017).

Correr é uma habilidade locomotora que envolve deslocamento do corpo através de contato com os pés no solo de uma forma rápida. Comumente

[...] a criança tenta correr por volta dos 18 meses, mas não há fase de voo (fase na qual o corpo é transportado por via aérea). As tentativas iniciais de correr assemelham-se a uma caminhada rápida. Uma fase de voo, assinalando a corrida geral, geralmente aparece entre o segundo e o terceiro aniversário (GALLAHUE; DONNELLY, 2008, p. 448).

Assim sendo, os sujeitos tiveram que realizar uma corrida de um ponto a outro, totalizando 20 metros. Também foi avaliada a melhor de duas execuções.

É considerado desempenho "excelência" $<=3,50 \mathrm{seg}$. para 8 anos e $<=3,00 \mathrm{seg}$. para 13 anos; e "fraco" > 4,47 seg. para 8 anos e > 3,81 para 13 anos. Idem ao teste anterior, todo o grupo atingiu desempenho insatisfatório e não houve nenhuma complicação na aplicação do mesmo.

Nota-se que de acordo com as tabelas normativas do PROESP-BR, que classifica o desempenho dos avaliados em "fraco", "razoável", "bom", "muito bom" e "excelência", divididos por faixa etária e sexo, todos os sujeitos da pesquisa obtiveram resultado considerado "fraco" em ambos os testes. Estes resultados vão de encontro com o estudo de Rosa Neto et al. 
(2013), no qual a criança autista avaliada foi classificada (por intermédio de avaliação semelhante) com desenvolvimento motor "muito inferior", antes e após intervenção motora.

Segundo as observações realizadas nas aulas, os respectivos autistas chegaram com um repertório motor bem limitado, apresentando dificuldades em diversas habilidades, dentre elas o salto, o qual os mesmos não conseguiam executar corretamente. Durante o período de 10 meses verificados, segundo os registros, foi constatado evolução significativa nos três domínios: motor, com aquisição e aprimoramento de habilidades motoras básicas e específicas; cognitivo, com autocorreção de movimentos, melhor desempenho em atividades que demandam mais atenção e concentração, como as de motricidade fina, por exemplo; e afetivo, com melhora no comportamento social referente à obedecer regras e afeto e confiança com os professores.

No entanto, quando analisamos a área motora separadamente, pode-se observar que os alunos autistas possuem dificuldades em virtude de desde a infância apresentarem relacionamento social deficitário e uma compreensão singular das atividades e do mundo, sendo privados muitas vezes - pelas próprias crianças, professores e até família - de brincadeiras e tarefas primordiais para um melhor desenvolvimento das crianças, comprometendo o campo motriz e cognição desses indivíduos.

Deste modo, foi constatado que já a partir das primeiras aulas os alunos começaram a apresentar pequenas melhorias, tanto motoras, quanto cognitivas e sociais, que com o tempo foram se tornando mais significativas. Deve-se salientar também que a área gravemente afetada pelo autismo, a relação social (DSM-V, 2013), demonstrou avanço na interação aluno/professor. Mas, é um processo gradual e que envolve empenho e conhecimento das características do transtorno e de metodologias de ensino mais eficazes por parte dos profissionais. Portanto, os resultados dos testes não apresentam grande relevância comparado ao desenvolvimento adquirido pelos alunos nas aulas e pelas questões apresentadas anteriormente como sendo obstáculos nesse processo.

\section{Conclusão}

O número de diagnósticos de TEA vem crescendo exponencialmente pelo mundo e isso se deve pela maior quantidade de informações e avanços científicos, que dão suporte aos profissionais, conseguindo diagnose precisa e muitas vezes precoce. Com isso, surgem cada vez mais ações voltadas para esse público com o intuito de auxiliar no seu desenvolvimento. No entanto, se trata de uma síndrome complexa e repleta de incógnitas que ainda carece de investigação. 
Logo, a presente pesquisa busca contribuir com a literatura pertinente a educação física e outras áreas, no sentido de ampliar os conhecimentos no tocante à importância e necessidade de práticas corporais para pessoas no espectro autista.

Diante da falta de estudos na área da atividade física para autistas, vale ressaltar a urgência de se criar ferramentas específicas para se avaliar o estado motriz desses indivíduos, em vista do expressivo número de pessoas com essa condição inseridos na sociedade, assim como a urgência de mais capacitação para os profissionais que atuam na área. Deste modo, o exercício físico surge como proposta no campo do autismo, visando a inclusão desses sujeitos nas instituições de ensino e na sociedade através do corpo. Para isso, precisamos cada vez mais de professores de educação física escolar e atividade motora, empenhados e com conhecimento. A elaboração de políticas públicas mais eficazes também é um importante fator para que os autistas estejam inseridos nos meios sociais.

Apesar de os participantes alcançarem resultado "fraco" nos testes motores, é pertinente atentar-se para os efeitos de programas de atividade física para as pessoas com TEA. Como verificado na pesquisa, esse tipo de intervenção tem sido eficaz e benéfica para esses sujeitos, se tornando um interessante recurso de aperfeiçoamento motor, cognitivo e social, ao evidenciar a importância do profissional de educação física para as pessoas com essa e outras deficiências.

Através dos testes do PROESP e das observações das aulas, foi possível identificar que, mesmo não alcançando os resultados padrões para idade e sexo, a atividade física auxilia, não apenas o desenvolvimento motor, como favorece a melhora dos aspectos cognitivos e sociais do aluno autista. Vale salientar que o atraso motor verificado nesta e em outras pesquisas nos alertam para a inclusão nas aulas de educação física, necessitando que o professor ofereça atividades que não exijam altas habilidades esportivas e competitivas, a fim de que a turma possa participar e vivenciar os movimentos propostos de forma autônoma e cooperativa. $\mathrm{O}$ atraso motor constatado não pode restringir a participação dos autistas nas aulas de educação física, sejam elas na escola ou fora dela, visto que a atividade física conforme reforçam MedinaPapst e Marques (2010, p.37) é a base "para o desenvolvimento das diversas habilidades motoras básicas como andar, correr, saltar, galopar, arremessar e rebater”. Desse modo, acreditamos que a prática de atividade física tem papel importante na vida da população estudada.

\section{REFERÊNCIAS}

DSM-V. AMERICAN PSYCHIATRIC ASSOCIATION. Manual Diagnóstico e Estatístico de Transtornos Mentais. Tradução de Maria Inês Corrêa Nascimento. Porto Alegre: Artmed, 2014. $976 \mathrm{p}$. 
BRASIL. Constituição Federal de 1988. Brasília, 1988. Disponível em: <http://www.planalto.gov.br>. Acesso em: 27 maio 2017.

BRASIL. Lei de Diretrizes e Bases da Educação Nacional-Lei nº 9.394/96. Brasília, 1996. <http://www.planalto.gov.br>. Acesso em: 27 maio 2017.

BRASIL. Lei $\mathrm{n}^{\circ}$ 12.764, de 27 de dezembro de 2012. Brasília, 2012. <http://www.planalto.gov.br>. Acesso em: 27 maio 2017.

DIEHL, R. M. Qualificação científica da bateria de aptidão física de crianças e jovens com Deficiência Visual (BAF_DV). 2013. 162 f. Tese (Doutorado em Ciências do Movimento Humano) - Escola de Educação Física, Universidade Federal do Rio Grande do Sul, Porto Alegre, 2013.

FIORINI, M. L. S.; MANZINI, E. J. Dificuldades e Sucessos de Professores de Educação Física em Relação à Inclusão Escolar. Rev. Bras. Ed. Esp., Marília, v. 22, n. 1, p. 49-64, jan.-mar. 2016.

FIORINI, M. L. S.; MANZINI, E. J. Inclusão de Alunos com Deficiência na Aula de Educação Física: Identificando Dificuldades, Ações e Conteúdos para Prover a Formação do Professor. Rev. Bras. Ed. Esp., Marília, v. 20, n. 3, p. 387-404, jul.-set. 2014.

GADIA, C. A.; TUCHMAN, R.; ROTTA, N. T. Autismo e doenças invasivas de desenvolvimento. Jornal de Pediatria - v. 80, n. 2 (supl), 2004.

GALLAHUE, D. L.; DONNELLY, F. C. Educação Física Desenvolvimentista para Todas as Crianças. São Paulo: Phorte Editora, 2008. 725 p.

GAYA, A.; GAYA, A. PROJETO ESPORTE BRASIL: Manual. Disponível em: <https://www.proesp.ufrgs.br> Acesso em: 28 maio 2017.

GIL, A. C. Como elaborar projetos de pesquisa. São Paulo: Editora Atlas, 2002. 176 p.

KLIN, A. Autismo e síndrome de Asperger: uma visão geral. Revista Brasileira de Psiquiatria, Rio de Janeiro, 28 (Supl I): p. 3-11, 2006.

KRÜGER, G. R. et al. Atividades rítmicas e interação social em crianças com autismo: Efeitos de uma intervenção. Revista Portuguesa de Ciências do Desporto: Desporto e atividade física adaptada, Porto, s2, r2, 2016 b.

KRÜGER, G. R. et al. Barreiras à prática da atividade física em crianças com autismo de uma cidade de médio porte do sul do Brasil. Revista Portuguesa de Ciências do Desporto: Desporto e atividade física adaptada, Porto, s2, r2, 2016a.

LOURENÇO, C. C. V. et al. A eficácia de um programa de treino de trampolins na proficiência motora de crianças com transtorno do espectro do autismo. Rev. Bras. Ed. Esp., Marília, v. 22, n. 1, p. 39-48, jan.-mar., 2016.

MARQUES, A. C. O perfil do estilo de vida de pessoas com Síndrome de Down e normas para a avaliação da aptidão física. 2008. 162 f. Tese (Doutorado em Ciências do Movimento Humano) - Escola de Educação Física, Universidade Federal do Rio Grande do Sul, Porto Alegre, 2008.

MEDINA-PAPST, J.; MARQUES, I. Avaliação do desenvolvimento motor de crianças com dificuldades de aprendizagem. Revista Brasileira de Cineantropometria e Desempenho Humano, Florianópolis, 12 (1): p. 36-42, 2010. 
POETA, L. S.; ROSA NETO, F. Intervenção motora em uma criança com Transtorno do Déficit de Atenção/Hiperatividade (TDAH). Lecturas Educación Física y Deportes, Buenos Aires, n.89, ano 10, 2005.

ROSA NETO, F. et al. Efeitos da intervenção motora em uma criança com Transtorno do Espectro do Autismo. Temas sobre Desenvolvimento, v. 19, p. 110-114, 2013.

ROSA NETO, F. Manual de avaliação motora. Porto Alegre: Artmed, 2002. 144 p.

SOARES, A. M.; CAVALCANTE NETO, J. L. Avaliação do comportamento motor em crianças com Transtorno do Espectro do Autismo: uma revisão sistemática. Revista Brasileira de Educação Especial, Marília, v. 21, n. 3, p. 445-458, jul.-set. 2015.

SOUZA, J. R.; ASSIS, R. M. Alunos autistas nas aulas de educação física: limites e possibilidades do trabalho cotidiano. In: Encontro Estadual de Didática e Prática de Ensino (EDIPE) do Centro de Estudos e Pesquisas em Didática (CEPED), 6. Goiânia. Anais... Disponível em:

<http://www2.unucseh.ueg.br/ceped/edipe/anais/viedipe/PDF/GT6\%20Ed\%20Fisica\%20pdf/ GT6_ALUNOS\%20AUTISTAS\%20NAS\%20AULAS\%20DE\%20EDUCACAO\%20FISICA .pdf $>$. Acesso em: 22 fev. 2017.

Artigo recebido em: 07/03/2018

Artigo aceito em: 26/05/2018 